\title{
Surveillance des expositions en laboratoire aux agents pathogènes humains et aux toxines : Canada 2018
}

\author{
D Choucrallah'1, L Sarmiento', S Ettles ${ }^{1}$, F Tanguay ${ }^{1}$, M Heisz $^{1}$, E Falardeau ${ }^{1 \star}$
}

\section{Résumé}

Contexte : Le système de surveillance de déclaration des incidents en laboratoire au Canada (DILC) fait le suivi des incidents en laboratoire en vertu de la Loi sur les agents pathogènes humains et les toxines. L'année 2018 représente la troisième année complète de données.

Objectif : Décrire les incidents d'exposition en laboratoire et les infections contractées en laboratoire survenus au Canada en 2018 par rapport aux années précédentes puis, selon le secteur, les agents pathogènes humains et les toxines en cause, le nombre de personnes touchées, le type d'incident et les causes fondamentales.

Méthodologie : Les incidents en laboratoire qui sont survenus en 2018 ont été signalés par le biais du système DILC. Après une comparaison du nombre d'incidents en laboratoire, de personnes exposées et d'infections contractées en laboratoire avec les chiffres des années précédentes, les incidents ont fait l'objet d'une analyse par secteur, agent pathogène humain ou toxine en cause, type d'incident, personnes exposées, voie d'exposition et causes fondamentales. Le programme Microsoft Excel 2016 a été utilisé pour les statistiques descriptives.

Résultats : En 2018, il y a eu 89 incidents d'exposition à des agents pathogènes humains et 235 personnes ont été exposées. Cinq infections contractées en laboratoire ont été soupçonnées et une a été confirmée. Ce nombre d'incidents d'exposition équivaut à environ le double de celui signalé en $2017(n=44)$ et en $2016(n=46)$. Les secteurs universitaire et hospitalier ont connu le plus grand nombre d'incidents d'exposition, et le rapport sur l'incidence par permis se révélait le plus faible dans le secteur privé. La majorité des incidents ( $n=50 ; 56 \%$ ) concernaient des agents pathogènes humains du groupe de risque 2 qui étaient manipulés dans un laboratoire de niveau de confinement 2. Les expositions étaient principalement liées à des objets tranchants et pointus ou à des procédures, et les personnes les plus souvent exposées étaient des techniciens de laboratoire. Les principales causes fondamentales étaient l'interaction humaine et les procédures opérationnelles normalisées.

Conclusion : Malgré que, dans l'ensemble, l'incidence annuelle d'expositions en laboratoire demeure relativement faible au Canada, elle a augmenté en 2018 par rapport aux années antérieures. Nous ignorons en ce moment s'il s'agit d'une augmentation réelle de l'incidence ou d'une hausse des signalements, puisque l'établissement des estimations de référence est toujours en cours.
Cette oeuvre est mise à la disposition selon les termes de la licence internationale Creative Commons Attribution 4.0

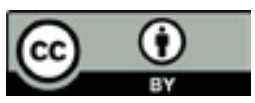

Affiliation

${ }^{1}$ Centre de la biosûreté, Agence de la santé publique du Canada, Ottawa, Ontario

\section{*Correspondance:}

emilie.falardeau@canada.ca

Citation proposée : Choucrallah D, Sarmiento L, Ettles S, Tanguay F, Heisz M, Falardeau E. Surveillance des expositions en laboratoire aux agents pathogènes humains et aux toxines : Canada 2018. Relevé des maladies transmissibles au Canada 2019;45(9):268-76. https://doi.org/10.14745/ccdr.v45i09a04f

Mots-clés : expositions en laboratoire, incidents de laboratoire, infections contractées en laboratoire, agents pathogènes humains et toxines, surveillance, déclaration des incidents en laboratoire au Canada, Centre de biosûreté 


\section{Introduction}

Les travaux de laboratoire avec des agents pathogènes humains et des toxines comportent un risque $d$ 'incidents susceptible de causer des expositions à la suite d'une libération accidentelle ou délibérée. Ces expositions peuvent toucher les personnes directement impliquées dans l'incident ou qui se trouvent à proximité. Elles pourraient éventuellement devenir une menace pour la santé publique, en cas de transmission dans la collectivité. Des rapports sur les incidents d'expositions soumis dans des délais raisonnables constituent un élément essentiel à la prévention d'éventuelles éclosions, puisqu'ils permettent d'agir rapidement en réponse aux expositions détectées.

Ces dernières années, le public a manifesté de plus en plus d'inquiétude à propos des possibilités de pandémie occasionnées par des infections contractées en laboratoire, puisqu'un plus grand nombre de pays autorisent la réalisation d'études sur le gain de fonction dans lesquelles les chercheurs accroissent la transmissibilité et la virulence d'agents pathogènes comme le virus de l'influenza $(1,2)$. Disposer d'un programme rigoureux de biosécurité et de biosûreté est impératif pour atténuer ces inquiétudes.

Le Centre de biosûreté de l'Agence de la santé publique du Canada a le mandat de prévenir les risques pour la santé publique occasionnés par l'utilisation d'agents pathogènes humains et de toxines au Canada, de les détecter et d'agir face à ces risques. Le Centre de biosûreté surveille les activités menées conformément à la Loi sur les agents pathogènes humains et les toxines (LAPHT) et au Règlement sur les agents pathogènes humains et les toxines (RAPHT). En décembre 2015, le Centre a mis sur pied un système de surveillance national, la déclaration des incidents de laboratoire au Canada (DILC), visant le signalement obligatoire des incidents de laboratoire qui comportent des agents pathogènes humains et des toxines. Les installations qui exercent des activités réglementées avec des agents pathogènes humains et des toxines doivent détenir un permis. Un seul permis peut couvrir plusieurs zones de confinement, mais ne pas couvrir différents groupes de risque (GR). La zone de confinement peut comprendre des zones normales réservées au travail de laboratoire, les zones de production de vaccins, voire des animaleries. Les installations détentrices de permis déterminent la manière de configurer le cadre de leurs permis en fonction de ces exigences. Dès l'enregistrement d'un permis, les installations doivent ellesmêmes déterminer le secteur singulier qui les représente le mieux en termes de structure organisationnelle. Les choix de secteur sont : académique, hospitalier, industrie/entreprise privée, santé publique ou autre gouvernement.

En vertu de la LAPHT et du RAPHT, les installations qui exercent des activités réglementées avec des agents pathogènes humains et des toxines doivent informer le Centre de biosûreté de
I'Agence de la santé publique du Canada sans délai de tout incident de laboratoire dans lequel des agents pathogènes humains et des toxines du GR2 ou plus sont en cause (3). Le Centre de biosûreté intervient sur-le-champ, notamment en faisant un suivi rapide. Il pourrait par exemple s'agir d'avis en matière de biosécurité et d'autres alertes ayant trait à de nouvelles tendances décelées et à de possibles habitudes inquiétantes, pour une amélioration continue de la biosécurité et de la biosûreté au Canada.

Les notifications soumises au système DILC peuvent comprendre des incidents avec ou sans exposition. Un incident de laboratoire pourrait concerner une exposition potentielle ou réelle à un agent biologique, que celle-ci cause ou non une infection contractée en laboratoire. Un incident sans exposition pourrait comprendre la possession, la production ou la libération accidentelle d'un agent pathogène ou d'une toxine; la disparition, la perte ou le vol d'un agent pathogène ou d'une toxine; la non-réception d'un agent biologique à cote de sécurité élevée dans les 24 heures suivant l'heure d'arrivée prévue (4). Afin d'optimiser la déclaration de ces incidents et de veiller au respect de la LAPHT, le Centre de biosûreté procède systématiquement à des activités de promotion, de surveillance et de vérification de la conformité. II rapporte également chaque année des renseignements sur les incidents de laboratoire afin de stimuler l'échange d'information, d'accroître la sensibilisation et d'encourager les signalements $(5,6)$.

La présente étude propose un sommaire descriptif des incidents de laboratoire qui sont survenus au Canada en 2018, en se concentrant sur les données relatives aux expositions et aux infections contractées en laboratoire. Ce rapport compte ainsi comparer brièvement les incidents d'exposition aux données des années antérieures et décrire les expositions en laboratoire par secteur, agents pathogènes humains et toxines en cause, type d'incident, personnes exposées, voie d'exposition et causes fondamentales.

\section{Méthodologie}

\section{Source de données}

Le portail Biosûreté est la portion « externe » de la DILC qui facilite la notification et la déclaration des incidents de laboratoire grâce à la soumission de rapports de notification et d'un ou plusieurs rapport(s) de suivi. Un système de gestion des relations avec la clientèle sert de plateforme « interne " pour la capture des données DILC, qui sont ensuite exportées dans un fichier Excel aux fins d'analyse.

Pour les besoins du présent rapport, nous avons extrait du système de gestion des relations avec la clientèle les données sur les incidents de laboratoire qui sont survenus entre le 1 er janvier et le 31 décembre 2018. Le programme Microsoft Excel 2016 a été utilisé pour les statistiques descriptives. 
Les incidents d'exposition comprenaient ceux ayant le pouvoir de causer une infection/intoxication et ceux ayant mené à une infection confirmée ou suspectée contractée en laboratoire par des agents pathogènes humains et des toxines du GR2 au GR4 contenues dans la portée de la LAPHT ou du RAPHT. L'analyse excluait les doublons, de même que les incidents et les déclarations hors du cadre de la LAPHT ou du RAPHT, comme les incidents liés à des agents pathogènes humains du GR1 ou des agents pathogènes dans leur milieu naturel.

\section{Analyse}

Les données relatives aux déclarations soumises au DILC ont été extraites et exportées dans Microsoft Excel 2016. Le nombre total d'incidents par permis a d'abord été comparé aux données rapportées pour 2017 et 2016. Par la suite, les incidents de laboratoire ont été soumis à une analyse par secteur, agents pathogènes humains ou toxines en cause, type d'incident, personnes exposées, voie d'exposition et causes fondamentales. Une analyse qualitative réalisée durant les activités de surveillance et de vérification de la conformité a permis de repérer certaines tendances.

\section{Résultats}

En 2018, il y a eu 89 incidents d'exposition à des agents pathogènes humains et 235 personnes ont été exposées, en plus d'un cas confirmé d'infection contractée en laboratoire. Ce nombre d'incidents d'exposition signalés en $2018(n=89)$ équivaut à environ le double de celui de $2017(n=44)$ et de 2016 ( $n=46)(3,4)$. Le nombre de cas confirmés d'infections contractées en laboratoire reste le même $(n=1)$ (figure 1). Au 31 décembre 2018, il y avait 985 permis en vigueur; 461 (47\%) étaient détenus dans le secteur de l'industrie privée, 203 (21\%) dans le secteur hospitalier, 200 (20\%) dans le secteur académique, 94 (9\%) dans un autre secteur gouvernemental (autre que la santé publique) et 27 (3\%) dans celui de la santé publique. Dans l'ensemble, cela correspond à une hausse de $20 \%$ du nombre de permis en vigueur par rapport

Figure 1 : Incidents déclarés d'exposition et d'infections contractées en laboratoire confirmées et soupçonnées, et permis en vigueur, Canada, de 2016 à 2018

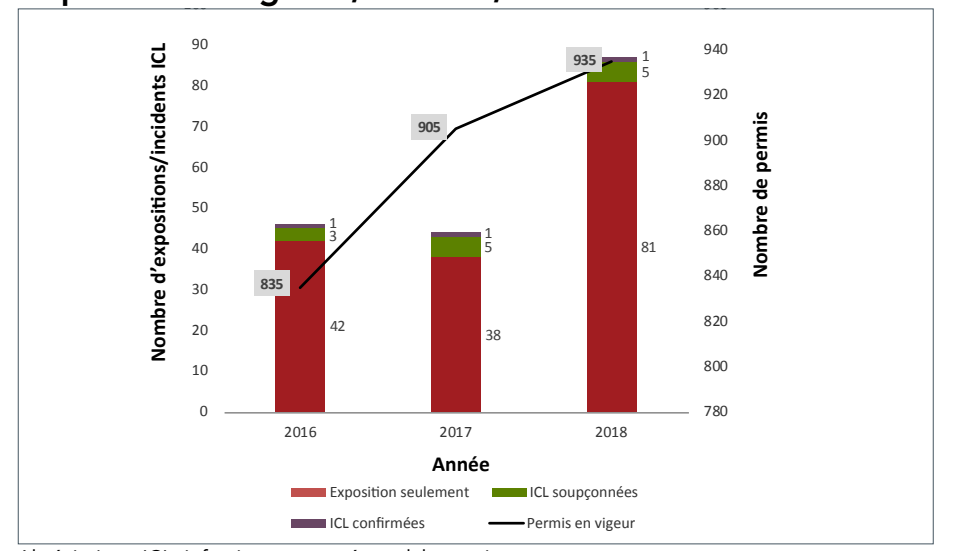

Abréviation : ICL, infection contractée en laboratoire

Sources : Les données de 2018 ont été extraites du système de déclaration des incidents de laboratoire au Canada (DILC). Les données de 2016 et 2017 proviennent des rapports publiés $(5,6)$ à $2016(n=835)$. Une augmentation du ratio entre incidents d'exposition et permis en vigueur apparaît en 2018 (1:11), comparativement à $2017(1: 20)$ et à 2016 (1:18) (données non illustrées).

\section{Incidents d'exposition par secteur}

En 2018, les secteurs académique ( $n=33 ; 37 \%$ ) et hospitalier ( $n=31 ; 35 \%$ ) ont déclaré le plus grand nombre d'incidents d'exposition (figure 2). Le secteur privé a affiché la plus forte proportion de permis en vigueur ( $n=461 ; 47 \%$ ) et une proportion relativement faible d'expositions déclarées $(n=8$; $9 \%$ ), ce qui mène au plus faible ratio entre incidents et permis $(1: 58)$. Le secteur de la santé publique affichait le plus fort ratio entre incidents et permis, avec 10 expositions déclarées et 27 permis en vigueur (1:3).

Figure 2 : Permis en vigueur et incidents d'exposition par secteur, Canada, 2018

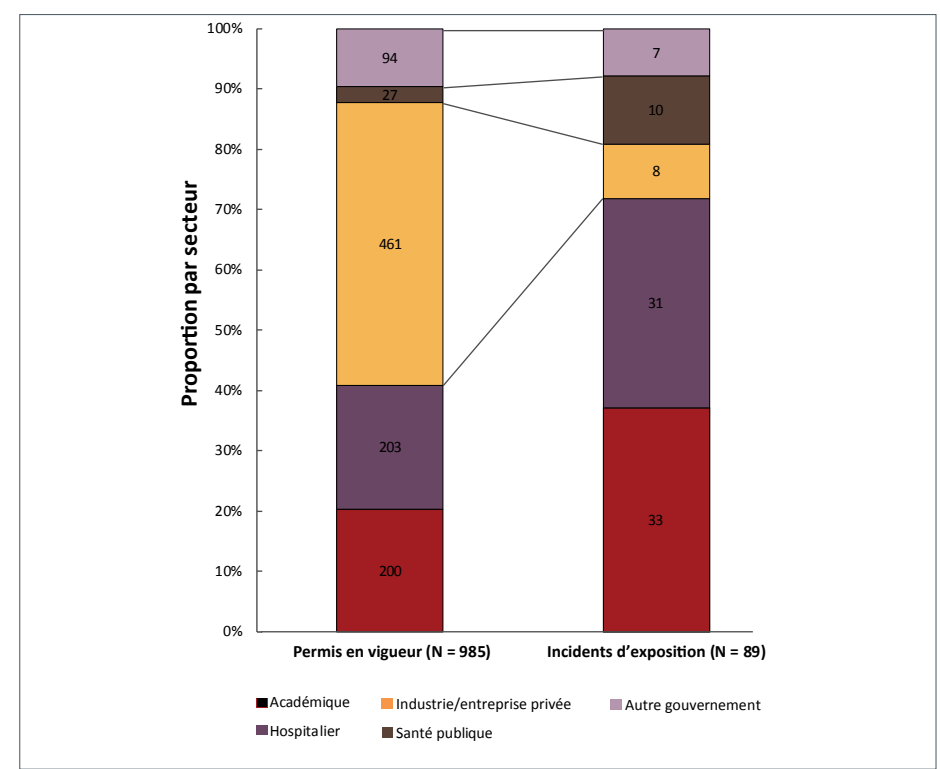

Remarques : Les installations détentrices de permis mentionnent elles-mêmes les secteurs Le secteur " académique " comprend notamment les universités, les collèges de médecine vétérinaire, les collèges et les cégeps [établissements et collèges techniques préuniversitaires québécois financés par l'État] et d'autres établissements d'enseignement; le secteur " hospitalier » regroupe les hôpitaux affiliés et non affiliés à une université; la catégorie " " industrie/entreprises privées » comprend les industries de la santé animale, de la santé humaine et de la biotechnologie, l'industrie pharmaceutique, I'industrie alimentaire, ainsi que les fournisseurs d'agents pathogènes et de toxines; le secteur de la " santé publique " comprend les laboratoires du gouvernement fédéral, des gouvernements provinciaux et territoriaux et des administrations municipales; les " autres secteurs gouvernementaux » regroupent les laboratoires vétérinaires ou de santé animale, les laboratoires environnementaux et d'autres laboratoires fédéraux, provinciaux, territoriaux et municipaux

Source : Déclaration des incidents de laboratoire au Canada (DILC)

La majorité des incidents d'exposition à des agents pathogènes humains et des toxines de la catégorie GR2 $(n=50)$ ont eu lieu dans le secteur académique ( $n=27 ; 54 \%$ ), suivi par le secteur hospitalier $(n=8 ; 16 \%)$ et le secteur privé $(n=7$; $14 \%$ ). Les incidents d'exposition à des agents pathogènes de catégorie GR3 ( $n=32$ ) sont survenus principalement dans le secteur hospitalier ( $n=20 ; 63 \%$ ), suivi par le secteur de la santé publique $(n=5 ; 16 \%)$ et le secteur académique $(n=4 ; 13 \%)$ (données non illustrées). 


\section{Agents pathogènes humains et toxines}

Des 89 incidents d'exposition, la majorité $(n=50 ; 56 \%)$ mettait en cause des agents pathogènes humains du GR2, 32 (36\%), des agents pathogènes du GR3 et un ( $1 \%$ ) une toxine (tableau 1). Un total de 18 (20\%) incidents mettaient en cause des agents biologiques à cote de sécurité élevée (ABCSE) de niveau GR3. Les agents biologiques les plus souvent en cause étaient des bactéries $(n=46)$, des virus $(n=17)$ et de champignons $(n=10)$.

Tableau 1 : Agents pathogènes humains et toxines en cause dans des incidents d'exposition, par niveau du groupe de risque, Canada, 2018

\begin{tabular}{|c|c|c|c|c|c|c|}
\hline \multirow{2}{*}{$\begin{array}{c}\text { Toxine } \\
\text { ou agent } \\
\text { pathogène }\end{array}$} & \multicolumn{2}{|c|}{ Non ABCSE } & \multicolumn{2}{|c|}{ ABCSE } & \multicolumn{2}{|c|}{ Total } \\
\hline & n & $\%$ & $n$ & $\%$ & $n$ & $\%$ \\
\hline Toxine $^{a}$ & 1 & 1 & - & - & 1 & 1 \\
\hline GR2 & 50 & 77 & - & - & 50 & 56 \\
\hline Bactérie & 30 & 46 & - & - & 30 & 34 \\
\hline Virus & 12 & 18 & - & - & 12 & 13 \\
\hline Parasite & 6 & 9 & - & - & 6 & 7 \\
\hline Prion & 2 & 3 & - & - & 2 & 2 \\
\hline GR3 & 14 & 22 & 18 & 100 & 32 & 36 \\
\hline Bactérie & 4 & 6 & 12 & 67 & 16 & 18 \\
\hline Virus & 5 & 8 & - & - & 5 & 6 \\
\hline Champignon & 4 & 6 & 6 & 33 & 10 & 11 \\
\hline Prion & 1 & 2 & - & - & 1 & 1 \\
\hline Inconnu & - & - & - & - & 6 & 7 \\
\hline Total & 65 & 100 & 18 & 100 & 89 & 100 \\
\hline
\end{tabular}

Abréviations : $G R$, groupe de risque; $A B C S E$, agent biologique à cote de sécurité élevée; -, sans objet

Les toxines ne sont pas catégorisées par groupe de risque. Les toxines qui sont inclus dans la portée du de la Loi sur les agents pathogènes humains et les toxines (LAPHT) sont énumérées dans l'annexe 1 de cette loi

Remarque : Les pourcentages sont arrondis au nombre entier le plus près

Source : Déclaration des incidents de laboratoire au Canada (DILC)

Les incidents d'exposition ont principalement eu lieu dans des laboratoires de niveau de confinement (NC) 2 ( $n=83 ; 93 \%$ ) et les autres, dans des laboratoires de NC3 $(n=6 ; 7 \%)$. La majorité des incidents survenus dans des laboratoires de NC2 mettaient en cause un agent pathogène du GR2 ( $n=50 ; 60 \%$ ) (figure 3).

Vingt-six (29\%) des incidents d'exposition découlaient de la possession accidentelle d'un agent biologique du GR3 dans un laboratoire de NC2 (figure 2). De ceux-ci, la moitié ( $n=13 ; 50 \%$ ) concernaient Brucella melitensis $(n=7)$ et Coccidioides immitis $(n=6)$ (tableau 2), et sont survenus principalement dans le secteur hospitalier $(n=11)$. Les expositions à Brucella spp ont atteint un sommet entre mars et novembre. Sept cas ont été signalés, la majorité étant survenue en juillet et en août. Le cas confirmé d'infection contractée en laboratoire mettait en cause Salmonella enterica subsp. enterica sérovar Enteritidis.
Figure 3 : Incidents d'exposition par niveau de confinement, et répartition des incidents $d$ 'exposition par groupes de risque dans des installations de niveau de confinement 2, Canada, 2018

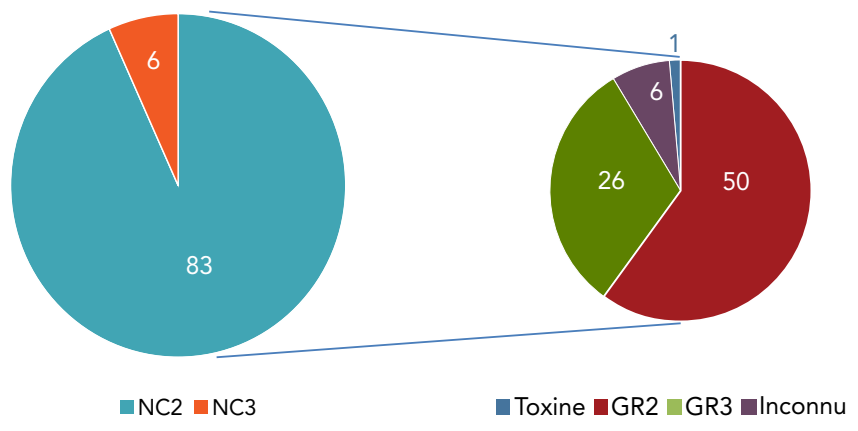

Abréviations : NC, niveau de confinement; GR, groupe de risque

Tableau 2 : Nombre d'incidents de laboratoire et de personnes exposées par agent biologique, Canada, 2018

\begin{tabular}{|c|c|c|c|c|}
\hline \multirow[b]{2}{*}{$\begin{array}{c}\text { Agent } \\
\text { biologique }\end{array}$} & \multirow[b]{2}{*}{$\begin{array}{l}\text { Incidents } \\
(\mathrm{N}=89)\end{array}$} & \multicolumn{3}{|c|}{ Personnes exposées $(N=235)$} \\
\hline & & $\begin{array}{c}\text { Exposition } \\
\text { seulement } \\
(n=229)\end{array}$ & $\begin{array}{l}\text { Personnes } \\
\text { exposées } \\
(n=235)\end{array}$ & $\begin{array}{c}\text { Confirmed } \\
\text { LAI } \\
(n=1)\end{array}$ \\
\hline RG2 & 50 & 63 & 4 & 1 \\
\hline $\begin{array}{l}\text { Neisseria } \\
\text { meningitidis }\end{array}$ & 5 & 8 & - & - \\
\hline $\begin{array}{l}\text { Staphylococcus } \\
\text { aureus }\end{array}$ & 3 & 3 & - & - \\
\hline Escherichia coli & 2 & 4 & - & - \\
\hline $\begin{array}{l}\text { Lymphocytic } \\
\text { choriomeningitis } \\
\text { mammarenavirus }\end{array}$ & 2 & 2 & 1 & - \\
\hline $\begin{array}{l}\text { Salmonella } \\
\text { enterica }\end{array}$ & 2 & 1 & - & 1 \\
\hline $\begin{array}{l}\text { Incidents avec } \\
\text { autres du GR2 }\end{array}$ & 36 & 45 & 3 & - \\
\hline GR3 & 32 & 159 & 1 & - \\
\hline $\begin{array}{l}\text { Brucella } \\
\text { melitensis }\end{array}$ & 7 & 105 & 1 & - \\
\hline $\begin{array}{l}\text { Coccidioides } \\
\text { immitis }\end{array}$ & 6 & 15 & - & - \\
\hline $\begin{array}{l}\text { Francisella } \\
\text { tularensis }\end{array}$ & 3 & 13 & - & - \\
\hline $\begin{array}{l}\text { Mycobacterium } \\
\text { tuberculosis }\end{array}$ & 3 & 9 & - & - \\
\hline $\begin{array}{l}\text { Incidents avec } \\
\text { autres du GR3 }\end{array}$ & 13 & 17 & - & - \\
\hline Toxines & 1 & 1 & - & -1 \\
\hline Inconnu & 6 & 6 & - & - \\
\hline
\end{tabular}

Abréviations : ICL, infection contractée en laboratoire; $G R$, groupe de risque; -, sans objet Source : Déclaration des incidents de laboratoire au Canada (DILC) 


\section{Types d'incident}

Les 89 expositions déclarées étaient liées à 110 types

d'incidents. Les types d'incidents les plus fréquents portaient sur des objets tranchants et pointus ( $n=26 ; 24 \%$ ) et des procédures $(n=23 ; 21 \%$ ) (figure 4). Un examen qualitatif des incidents d'exposition a permis de déceler une pointe d'incidents liés à du verre brisé. Des inspections sur place l'ont confirmée.

Figure 4 : Type d'incident d'exposition déclaré, Canada, $2018(\mathrm{~N}=110)$

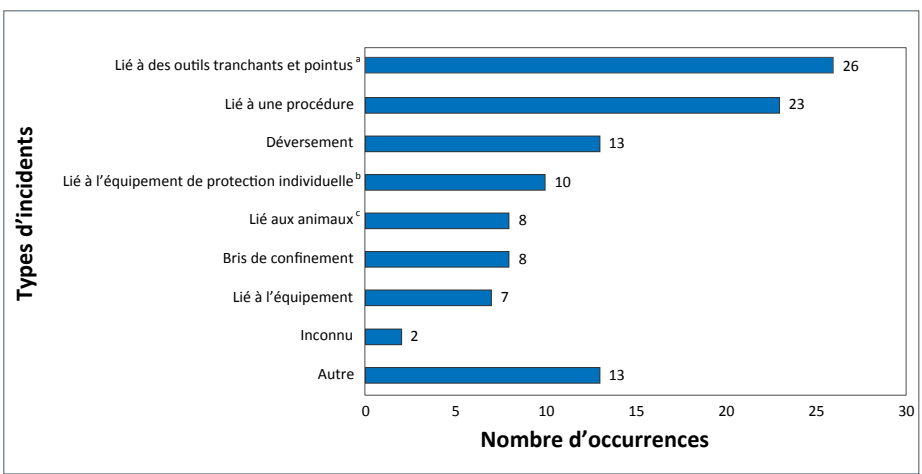

Remarque : La déclaration d'un seul incident peut comporter plus d'un type

a La catégorie "objets tranchants et pointus " comprend les piqûres d'aiguille et autres blessures par objets tranchants et pointus

"La catégorie " équipement de protection individuelle » comprend un équipement de protection individuelle inapproprié ou la défaillance d'un tel équipement

"La catégorie " animaux " comprend les morsures et les égratignures

Source : Déclaration des incidents de laboratoire au Canada (DILC)

\section{Personnes exposées}

Au cours des 89 incidents d'exposition, 235 personnes ont été exposées à un agent pathogène humain ou à une toxine. Des six infections contractée en laboratoire, cinq (2\%) aura été soupçonnées, alors qu'une seule $(0,4 \%)$ aura été confirmée. Les six cas d'infections contractées en laboratoire soupçonnées ou confirmée découlaient de l'exposition d'une seule personne par incident.

La majorité des incidents d'exposition ( $n=67 ; 75 \%$ ) concernait une seule personne exposée. Dix incidents (11\%) portaient sur l'exposition de 2 ou 3 personnes, et sept incidents (8\%), sur l'exposition de 4 à 10 personnes. II manquait des données dans deux déclarations. Les autres incidents $(n=3 ; 3 \%)$ signalaient l'exposition de 10 personnes ou plus; ceux-ci ont eu lieu dans le secteur hospitalier, où 14,29 et 53 personnes ont été exposées à Brucella melitensis.

Parmi les 235 personnes exposées, 39 (17\%) ont reçu les premiers soins et 85 (36\%), un traitement prophylactique dans les sept jours suivant l'incident. De plus, 8 (3\%) des personnes exposées ont reçu un traitement prophylactique après l'exposition plus de sept jours après l'incident. La plupart des personnes exposées étaient des techniciens de laboratoire ( $n=178 ; 76 \%$ ) ou des étudiants ( $n=22 ; 9 \%$ ) (figure 5 ).
Figure 5 : Rôles déclarés des personnes exposées, Canada, 2018 ( $\mathrm{N}=235$ )

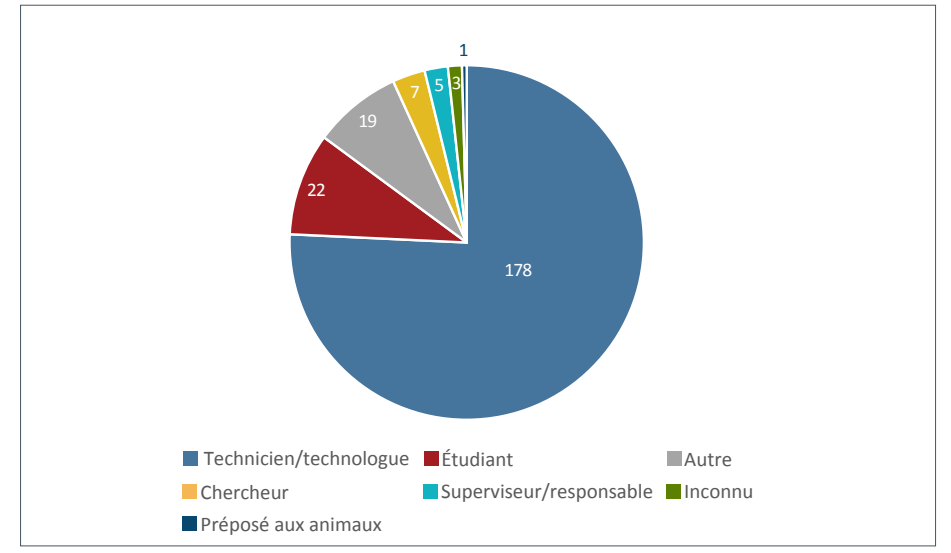

Source : Déclaration des incidents de laboratoire au Canada (DILC)

\section{Voie d'exposition}

Chez la plupart des 235 personnes exposées, l'exposition à des agents pathogènes ou à des toxines était survenue par inhalation ( $n=146 ; 62 \%$ ), tandis que l'inoculation/injection par piqûres $d$ 'aiguille ou objets tranchants et pointus arrivait au deuxième rang des voies d'exposition les plus fréquentes ( $n=28 ; 12 \%$ ) (figure 6).

Figure 6 : Voie d'exposition déclarée, Canada, 2018 $(\mathrm{N}=235)$

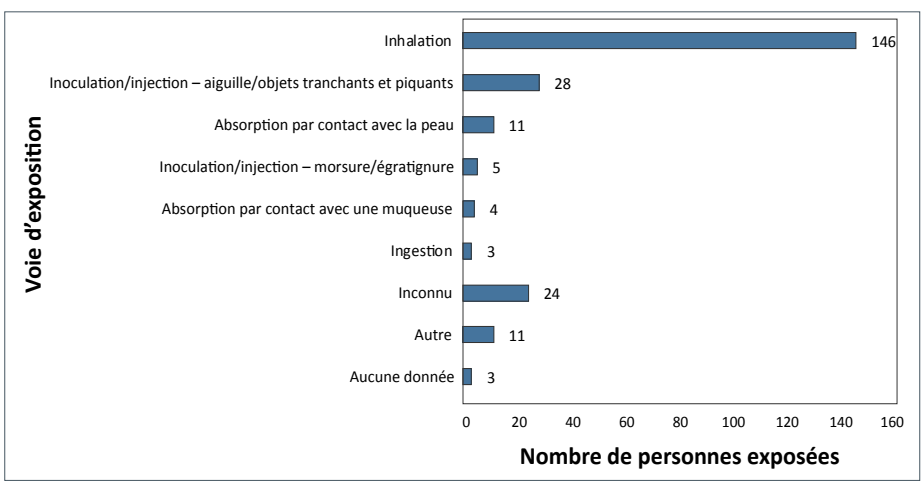

Source : Déclaration des incidents de laboratoire au Canada (DILC)

\section{Causes fondamentales et points à améliorer}

Les 89 incidents d'exposition déclarés en 2018 étaient associés à 233 causes fondamentales, ce qui correspond à 2,6 causes fondamentales par incident. Comme le montre le tableau 3, les interactions humaines et les manquements relatifs aux procédures opérationnelles normalisées se révélaient les principales causes fondamentales ( $n=53 ; 23 \%, n=50 ; 22 \%$ respectivement). 
Tableau 3 : Causes fondamentales et points à améliorer déclarés pour les incidents d'exposition à des agents pathogènes humains ou à des toxines, Canada, 2018 $(\mathrm{N}=233)$

\begin{tabular}{|c|c|c|c|}
\hline \multirow[t]{2}{*}{$\begin{array}{l}\text { Cause fondamentale/ } \\
\text { point à améliorer }\end{array}$} & \multirow[t]{2}{*}{$\begin{array}{l}\text { Exemple de point } \\
\text { préoccupant }\end{array}$} & \multicolumn{2}{|c|}{$\begin{array}{l}\text { Citations } \\
2018\end{array}$} \\
\hline & & $\mathbf{n}$ & $\%$ \\
\hline Interactions humaines & $\begin{array}{l}\text { Contraintes/pressions/ } \\
\text { exigences liées à la } \\
\text { charge de travail }\end{array}$ & 53 & 23 \\
\hline $\begin{array}{l}\text { Procédure opérationnelle } \\
\text { normalisée }\end{array}$ & $\begin{array}{l}\text { Procédures inconnues/ } \\
\text { pas suivies correctement }\end{array}$ & 52 & 22 \\
\hline Équipement & $\begin{array}{l}\text { Équipement mal conçu/ } \\
\text { entretenu }\end{array}$ & 32 & 14 \\
\hline Formation & $\begin{array}{l}\text { Formation non offerte ou } \\
\text { élaborée }\end{array}$ & 27 & 12 \\
\hline Communications & $\begin{array}{l}\text { Absence de méthode } \\
\text { ou de système de } \\
\text { communication }\end{array}$ & 24 & 10 \\
\hline Gestion et supervision & Supervision à améliorer & 24 & 10 \\
\hline Autre & Sans objet & 21 & 9 \\
\hline
\end{tabular}

\section{Discussion}

Dans l'ensemble, 89 incidents d'exposition ont mené à l'exposition de 235 personnes à des agents pathogènes humains et à des toxines. Parmi ces incidents, 5 (2\%) ont menés à une infection contractée en laboratoire soupçonnée et $1(0,4 \%)$ aura été confirmée. La majorité des incidents d'exposition est attribuable à des objets tranchants et pointus et à des manquements aux procédures. Les principales causes fondamentales étaient l'interaction humaine et le manque de connaissance ou de respect des procédures opérationnelles normalisées.

Même si l'incidence globale des expositions en laboratoire déclarées chaque année a doublé en 2018 par rapport aux deux années précédentes, nous ignorons encore s'il s'agit d'une hausse réelle de l'incidence ou d'une augmentation des déclarations puisque les estimations de référence ne sont pas encore établies. Le Centre de biosûreté apporte régulièrement des améliorations à son système de surveillance DILC afin de faciliter les déclarations et de préciser davantage les exigences réglementaires; il est possible que ces actions contribuent à l'augmentation des déclarations. Étant donné que le système de surveillance DILC est encore relativement nouveau, les organismes prendront sans doute un certain temps avant de s'habituer aux exigences réglementaires de déclaration.

Comme dans les années précédentes, les secteurs académique et hospitalier demeurent ceux affichant le plus grand nombre $d^{\prime}$ incidents $(5,6)$. II fallait s'y attendre, puisque la nature des travaux diffère entre les secteurs. Plus précisément, les laboratoires hospitaliers/diagnostiques ignorent souvent l'identité de l'agent biologique avec lesquels ils travaillent, ce qui accroît l'incertitude quant au statut du risque et la possibilité d'exposition quand le risque est sous-estimé. Les laboratoires du secteur privé travaillent habituellement sur des échantillons dans lesquels l'agent pathogène est déjà connu (p. ex. pour la mise au point de vaccins vivants).

Si les techniciens affichent le plus grand nombre d'expositions en laboratoire, cela pourrait s'expliquer par le fait qu'ils représentent la plus forte proportion de travailleurs manipulant des agents pathogènes humains et des toxines. Les techniciens œuvrent dans tous les secteurs, contrairement aux préposés aux animaux, par exemple. Parmi tout le personnel de laboratoire les étudiants se révélaient les victimes du plus grand nombre $d^{\prime}$ incidents d'exposition, après les techniciens. Des preuves anecdotiques indiquent que les étudiants du secteur académique ne connaîtraient ou ne respecteraient peut-être pas toutes les procédures de laboratoire et les mesures de sécurité, ce qui expliquerait le nombre élevé d'incidents d'exposition dans cette population.

Plusieurs grandes constatations de cette étude coïncident avec les résultats rapportés dans les ouvrages publiés : la majorité des infections contractées en laboratoire surviennent dans des laboratoires de NC2; les cas de déversements et ceux liés aux objets tranchants et pointus sont les types d'incidents les plus souvent déclarés; I'inhalation et l'inoculation constituent les principales voies d'exposition; les causes fondamentales les plus souvent déclarées sont l'erreur humaine et les problèmes liés aux procédures opérationnelles normalisées $(7,8)$. Les ouvrages publiés montrent également que l'exposition à une bactérie est plus fréquente que celle aux agents pathogènes $(9,10)$. Parmi les agents biologiques les plus souvent mis en cause dans les expositions et les infections contractées en laboratoire se trouvent plus précisément Brucella melitensis, Coccidioides immitis, Francisella tularensis et Mycobacterium tuberculosis (11-13).

\section{Forces et limites}

La principale force de cette étude repose sur le fait qu'elle se fonde sur une déclaration obligatoire et normalisée et intègre un processus d'examen comportant la validation des données autodéclarées. Ce volet du système de surveillance permet une déclaration rapide et systématique grâce à laquelle le Centre de biosûreté évalue les mesures correctives mises en place par les installations détentrices de permis - pour y déceler les facteurs de risque potentiels et diffuser l'information. À titre d'exemple, à la suite de la détection d'une pointe d'incidents avec Brucella spp, en 2018, un publipostage électronique à I'intention des installations a été distribué, afin d'accroître la vigilance en matière de biosécurité (Publipostage électronique sur la biosécurité et biosûreté liées aux agents pathogènes et aux toxines, incidents de laboratoire mettant en cause Brucella spp en 2018 - sommet observé en juillet et août 2018). De même, les personnes concernées ont reçu un avis à propos d'un risque potentiel d'expositions liées à des objets tranchants et 
pointus causés par du verre brisé, accompagné des techniques à appliquer pour atténuer le risque (Bulletin sur la biosécurité et la biosûreté des agents pathogènes et des toxines, Rapport détaillé du programme de déclaration des incidents de laboratoire au Canada (DILC) : exposition liée à du verre brisé, octobre 2018). Ainsi, ce programme de réglementation et de surveillance permet de détecter rapidement les risques courants et émergents et de diffuser l'information pour sensibiliser davantage les personnes concernées partout au Canada aux risques et aux meilleures stratégies d'atténuation.

La plus grande limite de cette étude est la possibilité que les données soient incomplètes, puisque dans certains cas, les incidents de laboratoire pourraient ne pas être décelés ou simplement ne pas être déclarés en raison d'un manque de connaissance des exigences ou d'une réticence à déclarer des incidents. Diverses publications continuent de s'attaquer à ce problème, comme la ligne directrice sur la notification et la déclaration en vertu de la LAPHT et du RAPHT, les bulletins et les avis de biosécurité, ainsi que les activités de surveillance et de la vérification de la conformité visant à encourager la déclaration et la conformité. En ce moment, nous ne disposons ni de données exactes sur le nombre d'installations détentrices de permis qui ne se conforment pas aux exigences de déclaration, ni du nombre de travailleurs dans les laboratoires. Cela complique la formulation de conclusions constructives sur l'importance des ratios de déclarations par secteur.

\section{Prochaines étapes}

Dans un certain nombre d'endroits, les activités du Centre de biosûreté pourraient bénéficier de données et d'analyses additionnelles. Par exemple, pour évaluer si les étudiants font face à un risque d'expositions accru, il serait judicieux d'identifier le nombre d'employés dans les laboratoires au Canada et d'examiner la proportionnalité d'étudiants par rôles et secteur. Des renseignements de ce genre permettraient au Centre de biosûreté de déceler plus facilement les risques et de mieux cibler les activités de sensibilisation et de promotion de la conformité.

\section{Conclusion}

Malgré une incidence annuelle d'expositions en laboratoire demeurant faible au Canada, cette dernière a augmenté en 2018 par rapport aux années antérieures. Nous ignorons s'il s'agit d'une hausse réelle de l'incidence ou d'une augmentation des déclarations, puisque les estimations de référence sont encore en voie d'établissement. Une analyse des expositions déclarées sert à éclairer et à modifier les normes et les lignes directrices en matière de biosécurité, pour une amélioration continue de la biosécurité au Canada.

\section{Déclaration des auteurs}

D. C. - Surveillance des incidents, analyse des données première version, rédaction, révision et édition

L. S. - Analyse des données - première version, rédaction, révision et édition

S. E. - Rédaction - révision et édition

F. T. et M. H. - Surveillance de l'incidence, rédaction - révision, édition et supervision

E. F. - Rédaction - révision, édition et supervision

\section{Conflit d'intérêts}

Aucun.

\section{Remerciements}

Nous tenons à remercier $C$. Evans pour son expertise et ses conseils, ainsi que les membres du personnel du Bureau des opérations de biosécurité et de bioconfinement pour leur expertise et la remise de données supplémentaires. Nous tenons aussi à faire part de notre reconnaissance à tous les titulaires de permis et aux agents de la sécurité biologique pour la soumission de rapports de qualité.

\section{Financement}

Ce travail a été réalisé grâce au soutien de l'Agence de la santé publique du Canada, dans le cadre de son mandat de base.

\section{Références}

1. Patterson AP, Tabak LA, Fauci AS, Collins FS, Howard S. Research funding. A framework for decisions about research with HPAI H5N1 viruses. Science 2013 Mar;339(6123):10367. DOI PubMed

2. National Institutes of Health. Notice announcing the removal of the funding pause for gain-of-function research projects. Washington (DC): National Institutes of Health; 2017 Dec 19 (Accédé 2019-05-25). https://grants.nih.gov/grants/guide/ notice-files/NOT-OD-17-071.html

3. Agence de la santé publique du Canada. Guide canadien sur la biosécurité, Deuxième édition. Deuxième édition. Ottawa (ON) : Gouvernement du Canada; 2016 Mars. 346 p. https://www.canada.ca/content/dam/phac-aspc/migration/ cbsg-nldcb/cbh-gcb/assets/pdf/cbh-gcb-fra.pdf

4. Agence de la santé publique du Canada. Guide canadien sur la biosécurité, Deuxième édition. Deuxième édition. Ottawa (ON) : Gouvernement du Canada; 2015 Mars. 168 p. https://www.canada.ca/content/dam/phac-aspc/migration/ cbsg-nldcb/cbs-ncb/assets/pdf/cbsg-nldcb-fra.pdf 
5. Bienek A, Heisz M, Su M. Surveillance des expositions en laboratoire aux agents pathogènes humains et aux toxines au Canada en 2016. Relevé des maladies transmissibles au Canada. 2017;43(11):259-68. DOI

6. Pomerleau-Normandin D, Heisz M, Tanguay F. Surveillance des expositions en laboratoire aux agents pathogènes humains et aux toxines au Canada en 2017. Relevé des maladies transmissibles au Canada 2018;44(11):337-44. DOI

7. Willemarck N, Van Vaerenbergh B, Descamps E, Brosius B, Dai Do Thi C, Leunda A. La-boratory-acquired infections in Belgium (2007-2012). Brussels: Institut Scienti-fique de Santé Publique Wetenschappelijk Instituut Volksgezondheid; 2015 Feb (Accédé 2019-05-25).

8. Pike RM. Laboratory-associated infections: incidence, fatalities, causes, and prevention. Annu Rev Microbiol 1979;33(1):41-66. DOl PubMed
9. Traxler RM, Lehman MW, Bosserman EA, Guerra MA, Smith TL. A literature review of laboratory-acquired brucellosis. J Clin Microbiol 2013 Sep;51(9):3055-62. DOI PubMed

10. Sewell DL. Laboratory-associated infections and biosafety. Clin Microbiol Rev 1995 Jul;8(3):389-405. DOI PubMed

11. Siengsanan-Lamont J, Blacksell SD. A review of laboratory-acquired infections in the Asia-Pacific: understanding risk and the need for improved biosafety for veterinary and zoonotic diseases. Trop Med Infect Dis 2018 Mar;3(2):36. DOI PubMed

12. Baron EJ, Miller JM. Bacterial and fungal infections among diagnostic laboratory workers: evaluating the risks. Diagn Microbiol Infect Dis 2008 Mar;60(3):241-6. DOI PubMed

13. Coelho AC, García Díez J. Biological risks and laboratory-acquired infections: a reality that cannot be ignored in health biotechnology. Front Bioeng Biotechnol 2015 Apr;3(56):56. DOI PubMed 


\section{Annexe}

Définitions liées à la Loi sur les agents pathogènes humains et les toxines (LAPHT)

\begin{tabular}{|c|c|}
\hline Terme & Définition \\
\hline Agent de la sécurité biologique (ASB) & $\begin{array}{l}\text { Personne désignée pour superviser les pratiques en matière de biosécurité et de biosûreté dans une } \\
\text { installation. }\end{array}$ \\
\hline Niveau de confinement (NC) & $\begin{array}{l}\text { Exigences minimales liées au confinement physique et aux pratiques opérationnelles visant la } \\
\text { manipulation sécuritaire d'agents pathogènes humains et de toxines dans les laboratoires. Il existe quatre } \\
\text { niveaux de confinement, qui vont du niveau de base (NC1) au niveau le plus élevé (NC4). }\end{array}$ \\
\hline Zone de confinement & $\begin{array}{l}\text { Espace physique qui répond aux exigences liées à un niveau de confinement donné. Il peut s'agir d'une } \\
\text { salle unique, d'une série de salles situées dans un même endroit ou d'une série de salles adjacentes. La } \\
\text { zone de confinement peut comprendre des zones réservées au soutien, y compris des antichambres, } \\
\text { équipés de douches, de vestiaires «propres » et de vestiaires « sales », le cas échéant. }\end{array}$ \\
\hline Exposition & $\begin{array}{l}\text { Contact ou proximité étroite avec des agents pathogènes humains ou des toxines pouvant } \\
\text { respectivement causer une infection ou une intoxication. Les voies d'exposition sont l'inhalation, } \\
\text { l'ingestion, l'inoculation et l'absorption. }\end{array}$ \\
\hline Formulaire de suivi de l'exposition & $\begin{array}{l}\text { Document utilisé pour rapporter et consigner des renseignements liés à une exposition accidentelle } \\
\text { préalablement déclarée à l'Agence de la santé publique du Canada, ainsi qu'à l'enquête qui y est } \\
\text { associée. }\end{array}$ \\
\hline $\begin{array}{l}\text { Formulaire de notification de } \\
\text { l'exposition }\end{array}$ & $\begin{array}{l}\text { Document utilisé pour déclarer une exposition accidentelle à l'Agence de la santé publique du Canada et } \\
\text { pour consigner les renseignements préliminaires associés à cette exposition. }\end{array}$ \\
\hline Incident & $\begin{array}{l}\text { Événement ou situation pouvant causer une blessure, un préjudice, une infection, une intoxication, une } \\
\text { maladie ou un dommage. Les incidents peuvent mettre en cause des matières infectieuses, des animaux } \\
\text { infectés ou des toxines. }\end{array}$ \\
\hline Laboratoire & $\begin{array}{l}\text { Espace réservé au sein d'une installation ou l'installation elle-même où des matières biologiques sont } \\
\text { manipulées à des fins scientifiques ou médicales. }\end{array}$ \\
\hline $\begin{array}{l}\text { Intoxication ou infection contractée en } \\
\text { laboratoire }\end{array}$ & $\begin{array}{l}\text { Intoxication ou infection résultant de l'exposition à des matières infectieuses, à des animaux infectés ou à } \\
\text { des toxines manipulés, ou entreposés dans la zone de confinement. }\end{array}$ \\
\hline Permis & $\begin{array}{l}\text { Autorisation délivrée par l'Agence de la santé publique du Canada en vertu de l'article } 18 \text { de la LAPHT, } \\
\text { permettant de mener une ou plusieurs activités réglementées comportant des agents pathogènes } \\
\text { humains ou des toxines. Un permis peut s'appliquer à de nombreuses zones de confinement. }\end{array}$ \\
\hline Groupe de risque (GR) & $\begin{array}{l}\text { Groupe dans lequel les matières biologiques sont classées en fonction de leurs caractéristiques } \\
\text { inhérentes, comme la pathogénicité, la virulence, le risque de propagation et l'existence d'un traitement } \\
\text { prophylactique ou thérapeutique efficace. Le groupe de risque énonce le risque pour la santé du } \\
\text { personnel et du public ainsi que la santé des animaux et des populations animales. }\end{array}$ \\
\hline $\begin{array}{l}\text { Agents biologiques à cote de sécurité } \\
\text { élevée (ABCSE) }\end{array}$ & $\begin{array}{l}\text { Sous-ensemble d'agents pathogènes humains et de toxines qui présentent un risque accru en matière } \\
\text { de biosûreté, en raison de la possibilité qu'on les utilise comme arme biologique. Les agents pathogènes } \\
\text { humains et les toxines sont décrits comme étant "précisés par le règlement »à l'article } 10 \text { du Règlement } \\
\text { sur les agents pathogènes humains et les toxines (RAPHT) Les agents biologiques à cote de sécurité } \\
\text { élevée comprennent donc tous les agents pathogènes des groupes de risque } 3 \text { et } 4 \text { figurant sur la Liste } \\
\text { des agents pathogènes humains ou animaux et des toxines réglementés à l'exportation, publiée par le } \\
\text { Groupe d'Australie et sujette à modifications, à l'exception du virus Duvenhage, du virus rabique et de } \\
\text { toutes les autres espèces du genre Lyssavirus, du virus de la stomatite vésiculaire, ainsi que du virus de } \\
\text { la chorioméningite lymphocytaire. Les agents biologiques à cote de sécurité élevée comprennent aussi } \\
\text { toutes les toxines qui se trouvent à la fois à l'annexe } 1 \text { de la LAPHT et sur la Liste des agents pathogènes } \\
\text { humains ou animaux et des toxines réglementés à l'exportation et qui sont présentes en quantités } \\
\text { supérieures aux quantités seuils énoncées au paragraphe } 10 \text { (2) du Règlement sur les agents pathogènes } \\
\text { humains et les toxines. }\end{array}$ \\
\hline
\end{tabular}

